\title{
Effects of Droplet Spacing on Evaporation of a Cluster of 13 Fuel Droplets
}

\author{
By Daisuke Segawa, Shinji NaKaya, Toshikazu Kadota, Go Agata, Dai Hara and Hiroyuki SugIHARA
}

Department of Mechanical Engineering, Osaka Prefecture University, Sakai, Japan

(Received May 2nd, 2008)

\begin{abstract}
The evaporation behavior of the central droplet in droplet clusters was observed in microgravity without combustion, and the effects of the droplet spacing were examined. The solidified-fuel fiber-suspension technique was utilized for preparing the monodispersed suspended-droplet cluster (MSDC) model. Most of the experiments were conducted with the HCP (hexagonal closest packing) structure cluster models of thirteen n-eicosane droplets at the atmospheric pressure, and some were conducted with the BCC (body-centered cubic) structure cluster models of nine 1-octadecanol droplets at an elevated pressure. The droplet images suggested that critical thermodynamic state was not attained at the ambient pressure above the critical pressure of the fuel, and the results at the elevated pressure were not largely different from those at the atmospheric pressure. The whole evaporation process was unsteady and the unsteadiness was enhanced with decreasing the droplet spacing. The initial heat-up time increased monotonically with decreasing the droplet spacing. The evaporation rate defined after the initial heat-up time was almost constant or showed an increasing trend with decreasing the droplet spacing.
\end{abstract}

Key Words: Liquid fuel, Droplet, Cluster, Evaporation, Microgravity

\section{Introduction}

In the chemical conversion of energy, combustion of liquid fuels plays an outstanding role. In most combustion devices for liquid fuels, such as boilers, diesel engines and gas turbine engines for example, spray combustion method is applied; Liquid fuels are first atomized into multiple fuel droplets, and they vaporize, ignite and burn. For improving thermal efficiency of those combustion devices and reducing carbon dioxide emissions, the detailed mechanism of spray combustion and droplet combustion should be elucidated. The deep understanding of spray combustion and droplet combustion would also help reducing the pollutant emissions from those combustion devices.

There have been many experimental studies on spray combustion and droplet combustion. Experimental studies on evaporation and combustion of isolated fuel droplets have provided a great deal of knowledge. On the basis of this knowledge, experimental studies have been conducted with arrays or matrices of fuel droplets. For experimental convenience, the droplet size is relatively large, and the droplet number is limited around ten ${ }^{1,2)}$. Larger droplets are preferred for better optical observation. On the other hand, quite large number of very fine fuel droplets is formed in fuel sprays ${ }^{3)}$. The droplets are unequally spaced and widely distributed, which prevents further understanding of local events. There is still lack of information on evaporation and combustion of droplet clusters with more than ten droplets, and additional experiments are needed.

We have started an experimental study on evaporation, autoignition and combustion of multiple droplet clusters. The monodispersed suspended-droplet cluster (MSDC) model with which arrangement, spacing and initial diameter of the droplet are well controlled has been developed. In the first phase of the experimental study ${ }^{4)}$, effects of the arrangement and the spacing on combustion characteristics of the droplet clusters which autoignited and burnt in a high-temperature air were examined. The initial diameter of each droplet was kept constant. For reducing the effects of the buoyant flow and simplifying the phenomena, the experiments were performed using microgravity environments in a drop shaft. The arrangement effect was examined by changing dimension or droplet number. The results showed that ignition delay and burning time increased with decreasing the droplet spacing regardless of the arrangement. The three-dimensional arrangement showed rather longer ignition delay and much shorter burning time than the two-dimensional arrangement. Larger droplet number resulted in longer ignition delay and longer burning time. The observation of the flame behavior of the autoignited droplet clusters offered an interesting feature, which would be relevant to Chiu's group combustion concept ${ }^{5-7)}$. Individual flames were formed around each droplet with the larger droplet spacing clusters, while group flame was formed with the smaller droplet spacing clusters. When the droplet spacing was in the intermediate range, a transition of flame mode occurred with the lapse of time, from the individual flames to the group flame.

In the second phase of the experimental study ${ }^{8)}$, effects of the initial droplet diameter as well as the droplet spacing on autoignition and early flame behavior of a spherical cluster of droplets in a high-temperature air were examined. The three-dimensional MSDC models of HCP (hexagonal closest packing) structure with 49 droplets were selected as the test clusters. The microgravity environment during the parabolic flight of aircraft, which provided rather longer microgravity 


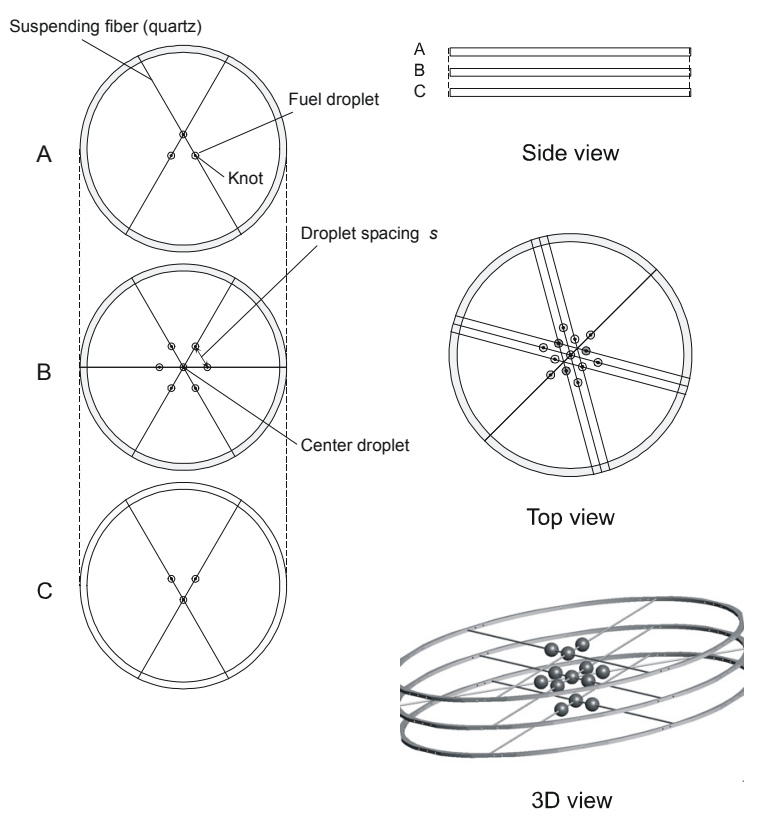

Fig. 1. MSDC model with 13 droplets

duration than drop shaft facilities, was utilized for the experiments with larger droplets. The flame immediately after ignition changed from the group flame to a cluster of the individual flames either with increasing the droplet spacing or decreasing the initial droplet diameter. Burning sphere diameter, a diameter of the spherical burning region, decreased at the beginning, and then increased. The transition from the individual flames to the group flame occurred around the time period at which the burning sphere diameter reached its minimum. The time period at which the burning sphere diameter reached its maximum was delayed and the expansion rate of the burning sphere was enhanced with decreasing the droplet spacing or with increasing the initial droplet diameter.

Droplet ignition is governed by transport processes as well as by chemical reactions. As the boiling point of the fuel was high and the droplet was large in our experiments, the mixture formation processes would be dominant in the autoignition. The mixture formation processes would also affect flame appearance immediately after ignition. In the present study, therefore, the evaporation behavior of the central droplet in droplet clusters was observed in microgravity without combustion, and the effects of the droplet spacing were examined. Most of the experiments were conducted with the clusters of 13 droplets at the atmospheric pressure, and some were conducted with the clusters of 9 droplets at an elevated pressure.

\section{Experimental Procedure}

\subsection{Droplet cluster}

The solidified-fuel fiber-suspension technique was utilized for preparing the monodispersed suspended-droplet cluster (MSDC) model. The fuel particles are held on quartz fibers in solid state at near room temperature, and therefore, arrangement, spacing and initial diameter of the fuel particles in the clusters are
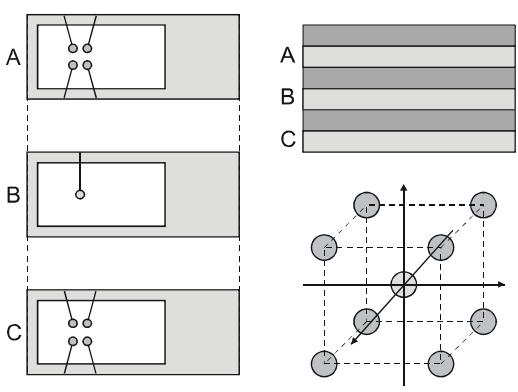

Fig. 2. MSDC model with 9 droplets

easily controlled. The cluster holders with the sample droplet clusters are prepared prior to the experiments. Rapid melting occurs just after the solidified fuel is subjected to high-temperature gaseous media, when the normal melting point of the fuels is little above room temperature and much lower than the gaseous media temperature ${ }^{9)}$.

The MSDC models with 13 n-eicosane droplets were tested at the atmospheric pressure. The normal melting point of n-eicosane is $310 \mathrm{~K}$. Figure 1 shows a schematic diagram of the MSDC model with 13 droplets. The cluster holder consisted of duralumin rings with $84 \mathrm{~mm}$ in inner diameter and $1.5 \mathrm{~mm}, 2 \mathrm{~mm}$ or $3 \mathrm{~mm}$ in height, suspending quartz fibers with diameter of $0.125 \mathrm{~mm}$ that spanned the duralumin ring, thin pillars and spacers of stainless steel, and a base plate with acrylic resin window. The duralumin rings were held on the three pillars, parallel to the base plate. The distances between the duralumin rings and the base plate were adjusted with the spacers. Each solidified droplet was held on the cross point of the quartz fibers or on the minute knot on the quartz fiber. Two-dimensional droplet arrangement was realized with the single ring and suspending quartz fibers. Neighboring three droplets were positioned at the apexes of an equilateral triangle. Three-dimensional droplet arrangement was realized with layers of the two-dimensional arrangement. Neighboring four droplets were positioned at the apexes of an equilateral tetrahedron. HCP (hexagonal closest packing) structure was, therefore, realized in the three-dimensional cluster. The droplet spacing $s$, the distance between neighboring droplets, was adjusted by arranging the quartz fibers and the knots on them and by changing the ring spacers.

The MSDC models with 1-octadecanol droplets were tested at an elevated pressure. Droplet number was reduced to nine due to smaller size of a furnace, in which the droplet cluster evaporated, and which were installed inside a high-pressure chamber. The normal melting point, the critical temperature and the critical pressure of 1-octadecanol are $331 \mathrm{~K}, 747 \mathrm{~K}$ and $1.41 \mathrm{MPa}$, respectively. Figure 2 shows a schematic diagram of the MSDC model with 9 droplets. The cluster holder consisted of duralumin frames, $1 \mathrm{~mm}$-thick plates with 30 $\mathrm{mm} \times 75 \mathrm{~mm}$ inner rectangle, suspending quartz fibers with diameter of $0.125 \mathrm{~mm}$ that cantilevered inward from the frame and shim plates. Each solidified droplet was held at the spherical tip of the quartz fiber. 


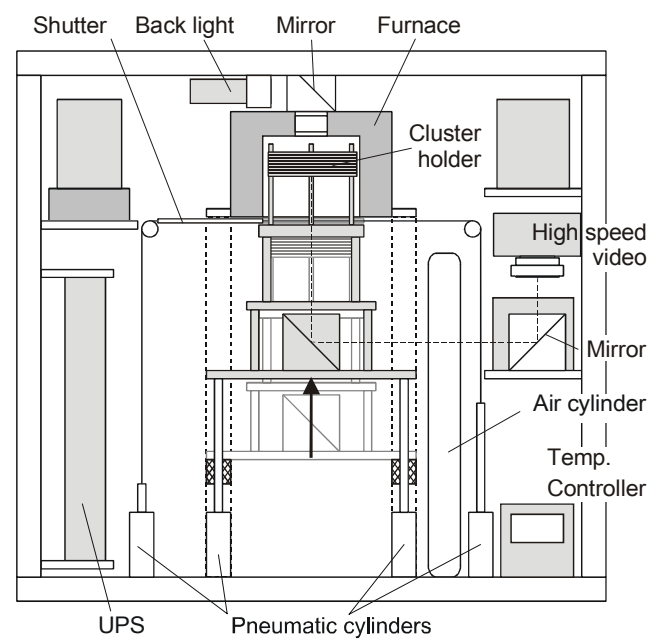

Fig. 3. Experimental setup for atmospheric pressure experiments

Two-dimensional droplet arrangement was realized with the single frame and suspending quartz fibers, and three-dimensional droplet arrangement was realized with layers of the two-dimensional arrangement. BCC (body-centered cubic) structure was realized in the three-dimensional cluster with 9 droplets. The 9 droplets were divided into two outer-droplet groups with 4 droplets and the central droplet, and the quartz fibers for each group were supported on one frame. The droplet spacing $s$ was adjusted by arranging the quartz fibers and by changing the shim plates.

The size of each droplet was checked with an enlarged backlight image. The projected image of the droplet was assumed to be a circle or an ellipse whose size was measured along major and minor axes perpendicular to each other. The diameter of the fuel droplet was defined as the diameter of the sphere with the same volume as the ellipsoidal body of rotation. The variations of the initial droplet diameter $d_{0}$ for the 13-droplet MSDC model and for the 9-droplet MSDC model were set to within 0.01 $\mathrm{mm}$ and within $0.05 \mathrm{~mm}$, respectively, on the basis of experimental convenience.

\subsection{Experimental setup and conditions}

Figure 3 shows a schematic diagram of the experimental setup for the atmospheric pressure experiments. The setup consisted of an electric furnace in which the 13-droplet MSDC model was allowed to evaporate, a sample transfer unit, a sequence controller that enabled the automatic operation of the apparatus, and optics. The electric furnace, $100 \mathrm{~mm}$ in inner diameter and $160 \mathrm{~mm}$ in inner height, was installed with quartz glass windows for optical access and with a shutter at its opening in the bottom wall for shielding thermal radiation from the electric furnace to the cluster on the sample transfer unit. A chromel-alumel type thermocouple with sheath diameter of $2.3 \mathrm{~mm}$ was used to measure and control the temperature inside the electric furnace. The sample transfer unit had a mounting base for the cluster holder and a mirror. The furnace shutter and the sample transfer unit were driven by a pneumatic system. Dampers reduced the impact at the arrival of the cluster holder to

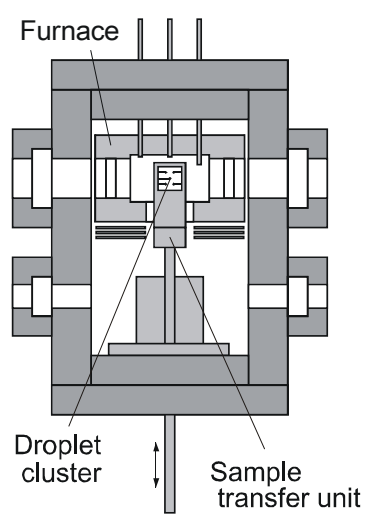

Fig. 4. High-pressure chamber for elevated pressure experiments

the experimental position inside the electric furnace. The optics had a couple of mirror, a lens unit, a backlight and a high-speed video camera. The evaporation process of the central droplet was observed by the camera at $125 \mathrm{fps}$ from the bottom opening of the electric furnace, with backlight illumination from the top opening of the electric furnace.

All the devices with a small-sized air cylinder for the pneumatic system were mounted on a falling assembly for a small drop tower in our laboratory. The cluster holder with the droplet cluster was set on the sample transfer unit after the furnace temperature was elevated. The furnace shutter was opened just before the start of the drop experiment, and the droplet cluster was quickly introduced into the electric furnace at the start. The temperature of the gaseous medium in the furnace was $670 \mathrm{~K}\left(=T_{\mathrm{a}}\right)$. The initial droplet diameter $d_{0}$ in the 13-droplet MSDC models was set to $0.45 \mathrm{~mm}$.

The experimental setup for the elevated pressure experiments consisted of the high-pressure chamber, the smaller electric furnace in which the 9-droplet MSDC model was allowed to evaporate, a sample transfer unit, a sequence controller that enabled the automatic operation of the apparatus, and optics. Figure 4 shows a schematic diagram of the high-pressure chamber. It was designed for the experiment under high-pressure conditions up to 10 $\mathrm{MPa}$. Two pairs of circular glass windows were located at the sidewall of the high-pressure chamber for optical access. The electric furnace with quartz glass windows occupied the upper portion of the high-pressure chamber. The furnace had the dimensions of $50 \mathrm{~mm}$ inner diameter and $70 \mathrm{~mm}$ inner height. A chromel-alumel type thermocouple with sheath diameter of $3.2 \mathrm{~mm}$ was used to measure and control the temperature inside the electric furnace. The sample transfer unit was installed below the furnace inside the high-pressure chamber. Thin aluminum plates were inserted between the furnace and the sample transfer unit for shielding thermal radiation from the furnace. A stepping motor installed outside the high-pressure chamber actuated a translating rod, which pushed up the sample transfer unit. The evaporation process of the droplet cluster was also observed by a high-speed video camera at $100 \mathrm{fps}$ with backlight illumination. 


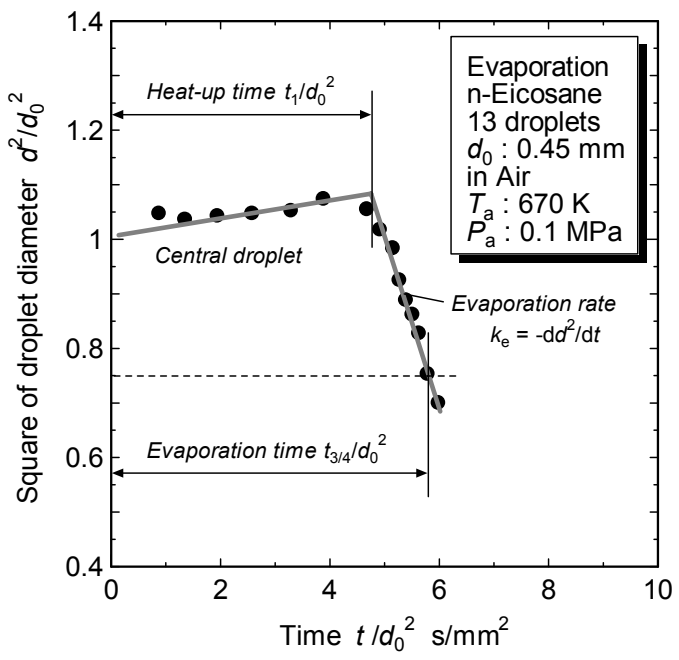

Fig. 5. Example of temporal variation of central droplet diameter squared at atmospheric pressure

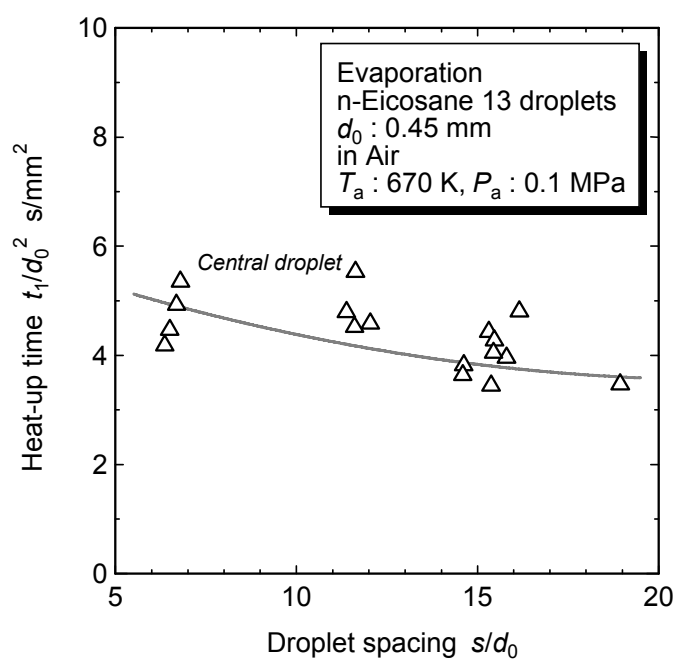

Fig. 6. Effect of droplet spacing on initial heat-up time at atmospheric pressure

All the devices were mounted on a falling assembly for a drop shaft facility. The cluster holder with the droplet cluster was set on the sample transfer unit and the chamber was charged with nitrogen before the drop experiment. After the furnace temperature was elevated, the drop experiment started. When the microgravity environment was attained, the droplet cluster was quickly introduced into the electric furnace. The reduced pressure $P_{\mathrm{r}}$, the pressure inside the chamber normalized by the critical pressure of the test fuel, was 2.0. The temperature of the gaseous medium in the furnace was $750 \mathrm{~K}\left(=T_{\mathrm{a}}\right)$. The initial droplet diameter $d_{0}$ in the 9-droplet MSDC models was set to $1 \mathrm{~mm}$.

\section{Results and Discussion}

\subsection{At atmospheric pressure}

Figure 5 shows an example of temporal variation of the

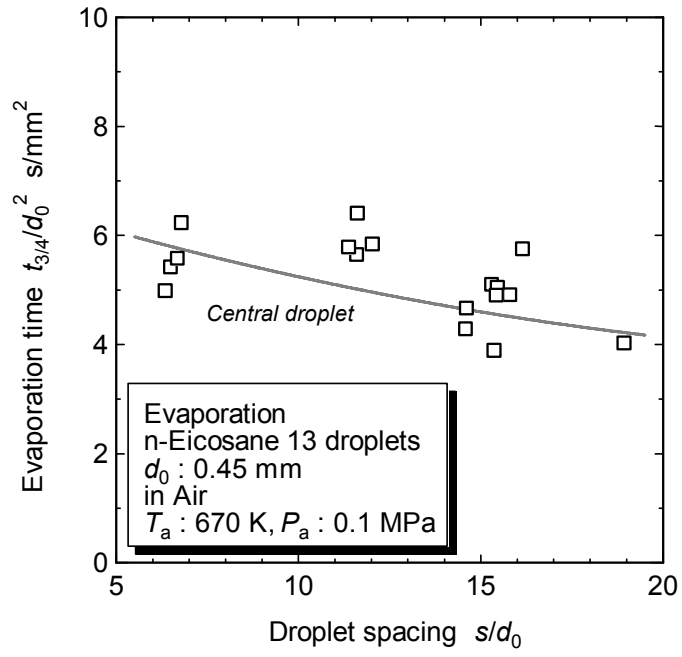

Fig. 7. Eeffect of droplet spacing on evaporation time to three-quarters of initial droplet diameter squared

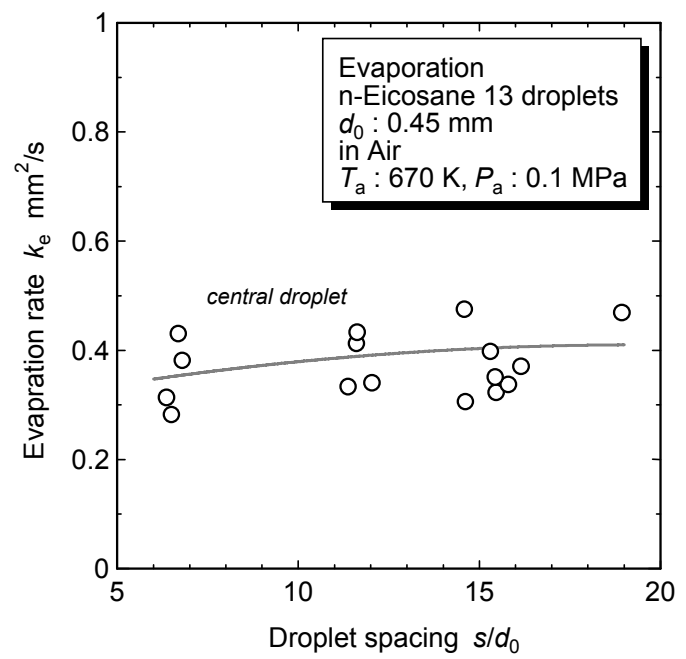

Fig. 8. Effect of droplet spacing on evaporation rate at atmospheric pressure

central droplet diameter squared. The droplet cluster was the 13-droplet MSDC model with the droplet spacing of 3 $\mathrm{mm}$. The ordinate is normalized by the square of the initial droplet diameter, and the abscissa is also divided by the square of the initial droplet diameter. The squared diameter of the central droplet gradually increased for a long time from the beginning, and then showed a peak and decreased very quickly. Two straight lines give good approximation for the temporal variation except at the beginning and near the peak, as shown in Fig. 5. The beginning term corresponds to the initial heat-up time $t_{1}$, and the latter term the rapid evaporation term after the droplet heat-up. The second straight line implies quasi-steady evaporation in the latter term, while the whole evaporation process would be unsteady. In the present study, the evaporation rate $k_{\mathrm{e}}$ was defined as the negative of the slope, $-\mathrm{d} d^{2} / \mathrm{d} t$, of the second straight line.

Figure 6 shows the effect of the droplet spacing on the 


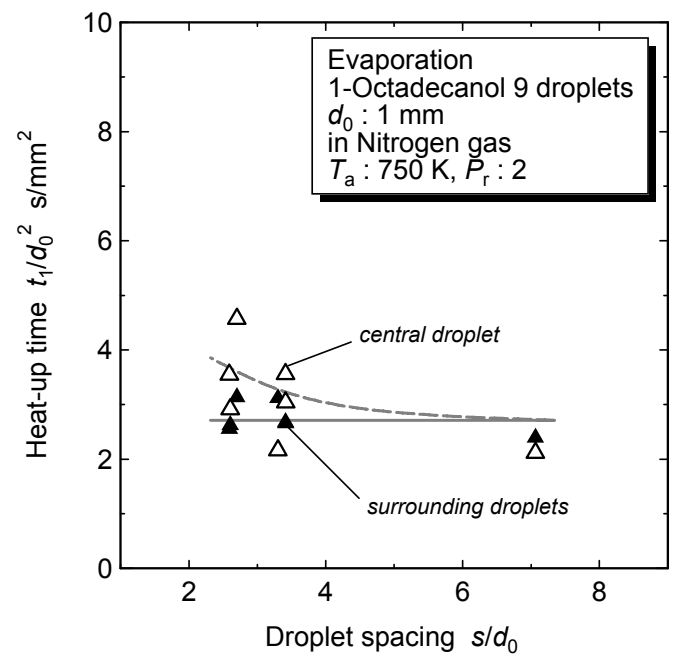

Fig. 9. Effect of droplet spacing on initial heat-up time at elevated pressure

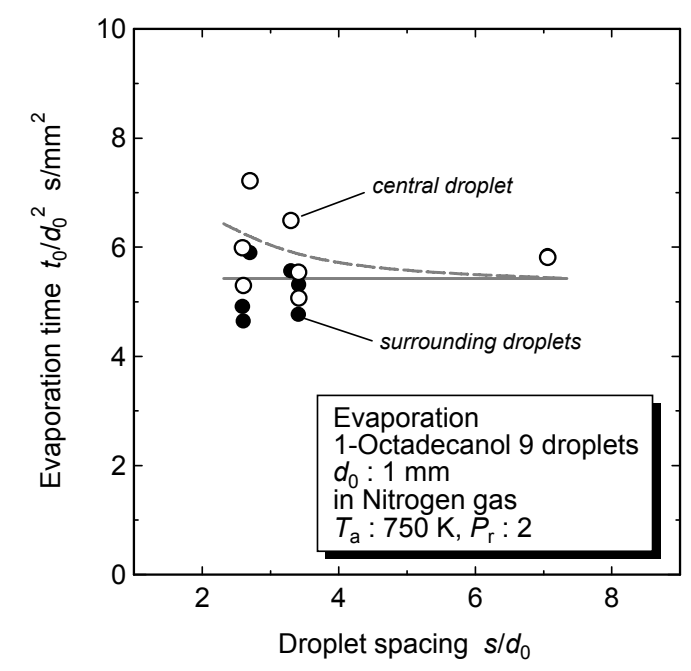

Fig. 10. Effect of droplet spacing on evaporation time at elevated pressure

initial heat-up time. The ordinate is divided by the square of the initial droplet diameter, and the abscissa is normalized by the initial droplet diameter. The initial heat-up time increased monotonically with decreasing the droplet spacing. The surrounding hot gaseous medium that supplied heat to the central droplet decreased with the droplet spacing, resulting in the prolonged heat-up time.

Figure 7 shows the effect of the droplet spacing on $t_{3 / 4}$, the evaporation time to three-quarters of the initial droplet diameter squared. The ordinate is divided by the square of the initial droplet diameter, and the abscissa is normalized by the initial droplet diameter. As the drop tower used for the experiment at the atmospheric pressure was too short to observe whole the evaporation event, the time period until the square of the droplet diameter decreased to three-quarters of the initial value was used as a measure of the evaporation time. The evaporation time $t_{3 / 4}$ also increased monotonically with decreasing the droplet

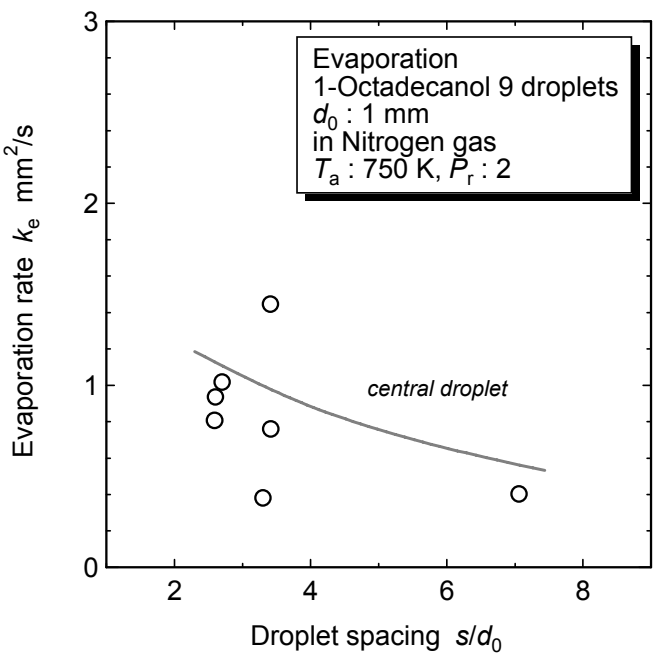

Fig. 11. Effect of droplet spacing on evaporation rate at elevated pressure

spacing. Figure 8 shows the effect of the droplet spacing on the evaporation rate. The abscissa is normalized by the initial droplet diameter. While smaller evaporation rate of the central droplet due to higher concentration of fuel vapor evaporated from the surrounding droplets was expected with smaller droplet spacing, the effect of the droplet spacing on the evaporation rate was not apparently observed. The initial heating of the central droplet in the droplet cluster would be affected by the surrounding droplets, resulting in the longer evaporation time.

\subsection{At elevated pressure}

The backlighted images of the droplet in the cluster were observed until the end of evaporation even at the ambient pressure above the critical pressure of the fuel tested. This suggests that the temperature of the droplets was lower than the critical temperature, and the critical thermodynamic state was be attained. The results were, therefore, not largely different from those at the atmospheric pressure.

Figure 9 shows the effect of the droplet spacing on the initial heat-up time. The droplet cluster was the 9-droplet MSDC model. The whole evaporation process was also unsteady, and the initial heat-up time was defined as the time period until the start of the quasi-steady evaporation. The ordinate is divided by the square of the initial droplet diameter, and the abscissa is normalized by the initial droplet diameter. The open circles correspond to the results for the central droplet and the solid circles the surrounding droplets. The initial heat-up time for the central droplet showed an increasing trend with decreasing the droplet spacing, while the initial heat-up time for the surrounding droplets was not largely affected by the droplet spacing.

Figure 10 shows the effect of the droplet spacing on the evaporation time $t_{0}$. The ordinate is divided by the square of the initial droplet diameter, and the abscissa is normalized by the initial droplet diameter. The trend was much the same as that for the initial heat-up time. Figure 11 shows the effect of the droplet spacing on the 
evaporation rate. The abscissa is normalized by the initial droplet diameter. Contrary to our expectation that was described previously, the evaporation rate showed an increasing trend with decreasing the droplet spacing. It is probable that higher concentration of fuel vapor evaporated from the surrounding droplets suppressed evaporation of the central droplet and continued to be heated-up. The central droplet could reach higher temperature, which enhanced evaporation after the end of evaporation of the surrounding droplets. Thermometry of the droplet clusters would be a future work.

\section{Conclusions}

The evaporation behavior of the central droplet in droplet clusters was observed in microgravity without combustion, and the effects of the droplet spacing were examined. The solidified-fuel fiber-suspension technique was utilized for preparing the monodispersed suspended-droplet cluster (MSDC) model. Most of the experiments were conducted with the HCP (hexagonal closest packing) structure cluster models of thirteen n-eicosane droplets at the atmospheric pressure, and some were conducted with the BCC (body-centered cubic) structure cluster models of nine 1-octadecanol droplets at an elevated pressure. Essential conclusions drawn from the present study are as follows;

- The droplet images suggested that critical thermodynamic state was not attained at the ambient pressure above the critical pressure of the fuel, and the results at the elevated pressure were not largely different from those at the atmospheric pressure.

- The whole evaporation process was unsteady and the unsteadiness was enhanced with decreasing the droplet spacing.

- The initial heat-up time increased monotonically with decreasing the droplet spacing.

- The evaporation rate defined after the initial heat-up time was almost constant or showed an increasing trend with decreasing the droplet spacing.

\section{Acknowledgments}

This study was supported in part by The Ministry of Education, Culture, Sports, Science and Technology, Japan, as Grant-in-Aid for Scientific Research (B). Many experimental devices including the high-pressure chamber were manufactured by Engineering Center at Osaka Prefecture University. Some experiments were conducted by Kajikawa, T. and Kin, T. The authors thank for the supports.

\section{References}

1) Kadowaki, T., Maruta, K., Kobayashi, H., Hasegawa, S. and Niioka, T.: Ignition Experiment on Droplet Matrix in Microgravity, Microgravity Sci. Technol., 9-4 (1996), pp. 269-274.

2) Nagata, H., Kudo, I., Ito, K., Nakamura, S. and Takeshita, Y.: Interactive Combustion of Two-Dimensionally Arranged Quasi-Droplet Clusters under Microgravity, Combust. Flame, 129 (2002), pp. 392-400.

3) Nomura, H., Koyama, M., Miyamoto, H., Ujiie, Y., Sato, J., Kono, M. and Yoda, S.: Microgravity Experiments of Flame Propagation in Ethanol Droplet-Vapor-Air Mixture, Proc. Combust. Inst., 28 (2000), pp. 999-1005.

4) Segawa, D., Yoshida, M., Nakaya, S. and Kadota, T.: Effects of Spacing and Arrangement of Droplet on Combustion Characteristics of Monodispersed Suspended-Droplet Cluster Model under Microgravity, Microgravity Sci. Tech., 17-3 (2005), pp. 23-30.

5) Chiu, H. H. and Liu, T. M.: Group Combustion of Liquid Droplets, Combust. Sci. Tech., 17 (1977), pp. 127-142.

6) Chiu, H. H., Kim, H. Y. and Croke, E. J.: Internal Group Combustion of Liquid Droplets, Proc. Combust. Inst., 19 (1982), pp. 971-980.

7) Chiu, H. H. and Lin, C. L.: Anomalous Group Combustion of Premixed Clusters, Proc. Combust. Inst., 26 (1996), pp. 1653-1661.

8) Segawa, D., Yoshida, M., Nakaya, S. and Kadota, T.: Autoignition and Early Flame Behavior of a Spherical Cluster of 49 Monodispersed Droplets, Proc. Combust. Inst., 31 (2007), pp. 2149-2156.

9) Segawa, D., Kajikawa, T. and Kadota, T.: Transcritical Phenomena of Autoignited Fuel Droplet at High Pressures under Microgravity, Microgravity Sci. Tech., 17-3 (2005), pp. 15-22. 